\title{
Diagnóstico de sensibilidad medioambiental en estudiantes universitarios
}

\section{Eugenia Mediavilla}

http://orcid.org/00oo-0002-5494-2779 Universidad Pablo de Olavide memedsos@acu.upo.es

\section{Silvia Medina Quintana}

https://orcid.org/0ooo-0oo2-7646-7534 Universidad de Córdoba smedina@uco.es

\section{Ignacio González López}

https://orcid.org/00oo-0002-9114-4370 Universidad de Córdoba ignacio.gonzalez@uco.es

\section{Resumen}

El artículo presenta un análisis de la dimensión afectiva, como parte de la conciencia ambiental, relativa a valores y creencias favorables al cuidado y respeto del ambiente. El estudio se configuró como un diseño descriptivo y no experimental, en el que se utilizó un cuestionario autoadministrado, con una muestra de 531 participantes, un $20 \%$ de la población objeto de estudio $(N=2051)$, integrada por la totalidad del alumnado de la Facultad de Ciencias de la Educación de la Universidad de Córdoba, España. Se encontró que existe un significativo nivel de conciencia ambiental, en términos de sensibilidad e interés por el estado actual del ambiente, y se confirmó la tendencia a la hipermetropía ambiental. Se concluye que es necesario pensar los problemas globales en su cotidianidad, bajando sus niveles de abstracción y, fundamentalmente, desde el lazo emocional que nos vincula con el ambiente.

\section{Palabras clave (Fuente: tesauro de la Unesco)}

Educación ambiental; enseñanza superior; España; formación de docentes; investigación pedagógica; Universidad de Córdoba. 


\title{
Diagnosing Environmental Awareness in University Students
}

\begin{abstract}
The article presents an analysis of the affective dimension as part of environmental awareness, meaning favorable values and beliefs about care and respect for the environment. The study has a descriptive, non-experimental design and employs a selfadministered questionnaire. The sample includes 531 participants, $20 \%$ of the studied population ( $N=2051)$ made up of the students from the School of Education Sciences of the Universidad de Córdoba, Spain. A significant level of environmental awareness (interest in the current state of the environment) was found. The trend towards environmental farsightedness was confirmed. In conclusion, it is necessary to think about global problems in its everyday nature, lowering their levels of abstraction, and fundamentally from our emotional bond with the environment.
\end{abstract}

\section{Keywords (Source: Unesco thesaurus)}

Environmental education; higher education; Spain; teacher education; educational research; Universidad de Córdoba. 


\section{Diagnóstico de sensibilidade meio ambiental em estudantes universitários}

Resumo

Este artigo apresenta uma análise da dimensão afetiva como parte da consciência ambiental, relativa a valores e crenças favoráveis ao cuidado e ao respeito do meio ambiente. Trata-se de estudo descritivo e não experimental, no qual foi utilizado um questionário autoadministrado, com uma amostra de 531 participantes, $20 \%$ da população-alvo do estudo $(N=2051)$, integrada pela totalidade de estudantes da Faculdade de Ciências da Educação da Universidad de Córdoba (Espanha). Verificouse que há significativo nivel de consciência ambiental em termos de sensibilidade e interesse pelo estado atual do ambiente, e confirmou-se a tendência à hipermetropia ambiental. Conclui-se que é necessário pensar sobre os problemas globais em sua cotidianidade, diminuindo seus niveis de abstração e, fundamentalmente, a partir do laço emocional que nos vincula com o meio ambiente.

\section{Palavras-chave (Fonte: tesauro da Unesco)}

Educação ambiental; ensino superior; Espanha; treinamento de profesor; pesquisa pedagógica; Universidad de Córdoba. 
$\mathrm{El}$ presente estudio se propone indagar y analizar una de las cuatro dimensiones de la conciencia ambiental, específicamente la dimensión afectiva, relativa a valores y creencias favorables al cuidado y respeto del ambiente que presenta el alumnado universitario de las titulaciones de la Facultad de Ciencias de la Educación (FCE) de la Universidad de Córdoba (UCO) en: Educación Infantil, Educación Primaria y Educación Social'.

La conciencia ambiental, entendida como un conjunto de conocimientos, experiencias y vivencias que las personas utilizan activamente en su relación con el ambiente (Febles et al., 2004), ha sido abordada en numerosas investigaciones. Desde los años setenta hasta la actualidad, sus diferentes formas de conceptuación, operacionalización y medición han sido objeto de estudio, derivado de un interés académico y social que no pierde vigencia (Báez, 2016; Brieger, 2019; Dietz et al., 1998; Dunlap y Jones, 2002; Stern, 2000; Van Liere y Dunlap, 1980).

Nuestro interés reside en realizar un diagnóstico de una de las dimensiones de la conciencia ambiental: la afectiva, en los y las estudiantes de magisterio, debido a: 1) el papel que se espera de estas personas en cuanto a su desempeño profesional como futuras maestras y maestros, partiendo de la idea de que el proceso educativo está atravesado por "una determinada estructura axiológica" que influencia a los educandos (Expósito, 2018, p. 311); y 2) la necesidad de continuar estudiando los factores que inciden en la posibilidad de transformación de la realidad socioambiental en determinados espacios, como lo es el educativo, que son clave en el proceso de enseñanza y socialización de valores y creencias ambientales, entre otros saberes significativos.

Resulta interesante también el contexto en donde se lleva a cabo la investigación, dado que, en primer lugar, en esta facultad dos titulaciones (Educación Infantil y Primaria) incorporan la educación ambiental

1 En el curso lectivo 2018-2019, posterior a la recolección de datos, se incorporó el grado de Psicología en la FCE de la UCO. como asignatura obligatoria y, en segundo lugar, existen campañas de comunicación y sensibilización ambiental dirigidas al alumnado como política ambiental de la universidad. Adicionalmente, la UCO forma parte de la Comisión Sectorial de Calidad Ambiental, Desarrollo Sostenible y Prevención de Riesgos (CADep) de la Conferencia de Rectores de las Universidades Españolas (CRUE), cuyo propósito es garantizar la incorporación de criterios de sostenibilidad en la enseñanza y el aprendizaje del alumnado, de modo que la sostenibilidad impregne todas las esferas de la docencia.

Este trabajo pretende realizar un aporte a la investigación en educación ambiental y generar información y herramientas orientadas a comparar y continuar midiendo las relaciones entre las personas y su medio, con la finalidad general de promover acciones relativas a la educación. La información puede ser utilizada tanto para el diseño de nuevos programas de sensibilización como para la evaluación de planes y acciones ya ejecutados en la materia, y para la generación y reformulación de contenidos de las asignaturas ambientales de las diferentes titulaciones universitarias. Se parte de que no es posible trabajar en el diseño, implementación o evaluación de determinados programas y proyectos del campo educativo, y en el ámbito de cualquier disciplina, sin un diagnóstico del estado de situación o punto de partida, para el desarrollo y la consecución de las metas y objetivos propuestos.

\section{Marco teórico}

\section{Conciencia ambiental}

La principal contribución al significado teórico de la conciencia ambiental ha sido realizada por Dunlap y colaboradores. Como referentes pioneros en materia de investigación sobre sociología ambiental en los Estados Unidos, estos autores comienzan a estudiar este concepto en la década de 1970, definiendo la misma como "el grado en que las personas son conscientes de los problemas relativos al 
ambiente como así también el grado de apoyo a los esfuerzos para resolverlos, y a la disposición a contribuir personalmente a su solución" (Dunlap y Jones, 2002, p. 485). Por su parte, Zelezny y Schultz denominan la conciencia ambiental como un conjunto de "factores psicológicos específicos que guían a las personas a llevar a cabo comportamientos proambientales" (2000, p. 367). Schultz (2001) sostiene que existen diferentes tipos de conciencia ambiental, de acuerdo con la manera en que las personas se relacionan con la naturaleza o, más específicamente, perciben las consecuencias de la degradación ambiental en función de la orientación de nuestros valores: en relación con nosotros mismos (orientación egoísta), con otras personas (orientación altruista) y con la biosfera (orientación biosférica). Esto significa que las personas empiezan a preocuparse por la problemática ambiental cuando ven amenazado su sistema de valores, ya sea que esté centrado en ellas mismas, en las demás o en cualquier ser con vida.

Otro de los esquemas conceptuales referentes en materia de conciencia ambiental es el propuesto por Corraliza et al., quienes la definen como "el conjunto de imágenes y representaciones que tienen por objeto de atención el medio ambiente o aspectos particulares del mismo, tales como la disminución de especies, la escasez de recursos naturales, la calidad ambiental en su conjunto, entre otros muchos temas" (2004, p. 106). Por su parte, Jiménez y Lafuente definen la conciencia ambiental como "determinados procesos asociados a las acciones que intentan reducir el impacto ambiental de la acción humana" (2006, p. 122), esto es, la forma de interpretar el mundo a través de constructos psicológicos, sean creencias, actitudes, valores y comportamientos, y de actuar en consecuencia en materia ambiental. En este mismo sentido, estos autores reafirman su definición anterior y consideran la conciencia ambiental como la dimensión actitudinal o psicológica del comportamiento proambiental (2010, p.732) entendiendo por tal toda acción que tenga la intención de cambiar el ambiente en beneficio del mismo (Stern, 2000).
De acuerdo con los aportes realizados por Chuliá (1995, p. 4), la conciencia ambiental puede conceptualizarse a partir de sus dimensiones cognitiva, afectiva, conativa y activa (que puede ser tanto colectiva como individual). La dimensión cognitiva se refiere al conjunto de conocimientos e información que poseen las personas en relación con la problemática ambiental. La dimensión afectiva agrupa el conjunto de sentimientos de preocupación por el estado del ambiente y el grado de adhesión a valores favorables al cuidado del medio ambiente. Por su parte, la dimensión conativa es definida como el grado de predisposición a actuar personalmente con criterios ecológicos y a aceptar los posibles costes que tienen las acciones a favor de la protección ambiental. Finalmente, la dimensión activa se caracteriza por la motivación y competencia en comportamientos individuales y/o colectivos y por hábitos de consumo que reivindican la defensa del ambiente.

\section{Dimensión afectiva de la conciencia ambiental}

La dimensión afectiva, nuestro objeto de estudio, se define como el grado de adhesión a creencias y valores afines al cuidado y respeto del entorno, así como el conjunto de sentimientos de preocupación por y estimación de la gravedad o importancia que se otorgan a los diferentes problemas ambientales. Al respecto, Báez sostiene que "la sensibilidad ambiental muestra cómo los problemas del medio conciernen al individuo, en calidad de interés, afectación, preocupación o receptividad hacia las problemáticas medioambientales" (2016, p. 369).

Se considera que el componente afectivo de la conciencia ambiental es de suma relevancia, ya que el conjunto de nuestras creencias y valores representa la manera de ver el mundo y configura nuestras experiencias de vida, pudiendo condicionar nuestras acciones, deseos y objetivos (Murray, 2011). Las creencias y valores ambientales cumplen un rol en nuestra forma de vincularnos con el medio, pudiendo influenciar efectivamente el compromiso con el 
comportamiento a favor del ambiente (Kaida y Kaida, 2015; Ortega y García, 2013; Schultz et al., 2005).

Las creencias son definidas como un conjunto de conceptos, opiniones, ideas, informaciones, percepciones y afirmaciones sobre la naturaleza de un fenómeno (Acebal, 2010). En particular, las creencias ambientales son estructuras que guían los procesos cognitivos y motivacionales y contribuyen a la comprensión de los valores, actitudes y comportamientos que tienen las personas en su relación con el ambiente. Las mismas pueden ser ecocéntricas y representar una visión integradora de las relaciones entre las personas y la naturaleza en la búsqueda del equilibrio socioambiental, o antropocéntricas, al considerar que los recursos naturales son inagotables y de uso ilimitado, que asocian al ambiente como sinónimo de naturaleza y a los problemas medioambientales como únicamente relativos a la biosfera, de modo que no tienen en cuenta otras especies y ecosistemas, aparte de los seres humanos (Pato et al., 2005).

Estas creencias están condicionadas por valores, los cuales son considerados constructos psicológicos abstractos que trascienden situaciones y acciones particulares y orientan la toma de decisiones, representando "metas deseables, transituacionales, variables en importancia, que sirven de principios rectores en la vida de la gente" (Schwartz y Barnea, 1995, p. 17). Otra manera de definirlos es como conjunto de juicios adquiridos a lo largo de nuestras vidas, que comenzamos heredando de personas cercanas pertenecientes al entorno familiar y de otras instituciones, tales como el sistema educativo, el sistema jurídico-político, el orden social, mandatos religiosos, y que terminamos de construir a partir de las experiencias personales que se van sucediendo a lo largo de la vida (Murray, 2011).

En sintesis, las creencias se configuran como informaciones sobre lo que la realidad es, mientras que los valores se configuran como el deber ser de la realidad. Ambos están interrelacionados, se justifican entre sí y se complementan, conformando los paradigmas o modos de ver y entender las realidades que se perciben. Por esta razón, "la jerarquización de los valores personales relacionados con el respeto al medio ambiente influye decisivamente en los sentimientos de preocupación y la estimación de gravedad de los diferentes problemas ambientales" (Gomera, 2011, p. 40).

En este sentido la dimensión afectiva de la conciencia ambiental también ha sido caracterizada por el conjunto de emociones y sentimientos relacionados con la temática medioambiental y que abarcan no solo la preocupación por el estado del ambiente, sino su percepción como medio de vida en el que se puede desarrollar un sentido de pertenencia y empatía con lo que nos rodea (Corraliza et al., 2004). Este sentido de comunidad implica un arraigo con la tierra y tiene también un papel cohesionador para las obligaciones comunitarias recíprocas entre sus habitantes (Manrique, 2019).

La autopercepción de esta dimensión de la conciencia ambiental está contenida por un marco emocional que la posibilita (Mora, 2019). Las emociones pueden definirse como un "componente esencial de nuestro modo de ser y estar en el mundo en tanto influencian nuestras percepciones y reacciones" (Brunel, 1995, p. 177) y en cuanto construcciones históricas en proceso que también pueden articular nuestros modos de saber (Fernández y Alegre, 2019). Los sentimientos son "el estado del sujeto caracterizado por la impresión afectiva que le causa determinada persona, animal, cosa, recuerdo o situación en general" y están relacionados con las experiencias subjetivas de las emociones (Castillo, 2008, p. 346).

Otro de los componentes de la dimensión afectiva es la hipermetropía ambiental, la cual refiere a la tendencia a una mayor preocupación por parte de las personas cuanto más alejado se encuentre un problema ambiental, al tiempo que el sentimiento de responsabilidad para poder resolver estos problemas disminuye con las distancias y acrecienta los sentimientos de impotencia (Uzzell, 2000). En otros términos, la conciencia sobre la problemática am- 
biental en un nivel de abstracción muy alto puede ser contraproducente, porque no necesariamente se traduce en actitudes personales que lleven a actuar frente a esa problemática (Chen, 2020).

La hipermetropía ambiental se debe a que el sistema de creencias ambientales de las personas es parte de un sistema mayor de creencias sociales que cataloga los problemas ambientales como globales por definición (García et al., 2005; Stern y Dietz, 1994). Este fenómeno parece reforzado, por un lado, por los grandes medios de comunicación, a través de la televisión, en mayor medida, y de los periódicos y redes sociales, que dan mayor cobertura a los problemas globales del medio ambiente en relación con los problemas considerados locales y, asimismo, por otro lado, por agrupaciones u organizaciones ecologistas internacionales con gran visibilidad mediática que promueven campañas relativas a fenómenos globales, tales como el calentamiento global, la desertización de los polos o la destrucción de la capa de ozono (García et al., 2005).

\section{Objetivos}

El presente estudio ha estado pensado para responder a distintos objetivos que han sido las guías de todo el proceso de investigación. De este modo, el objetivo general consistió en analizar la dimensión afectiva de la conciencia ambiental en el alumnado de la Facultad de Ciencias de la Educación de la Universidad de Córdoba. Para tal propósito, ha sido necesario ir respondiendo de un modo específico a las siguientes finalidades:

- Estudiar las creencias y valores medioambientales que presenta el alumnado de Ciencias de la Educación de la Universidad de Córdoba.

- Analizar los niveles de preocupación y sensibilidad medioambientales de la población sujeto de la investigación.

- Evaluar si el alumnado bajo estudio presenta niveles de hipermetropía ambiental.
- Detectar elementos clave que sirvan como información básica para llevar a cabo acciones relativas a la formación y especialización curricular en medio ambiente.

\section{Método}

La investigación se configuró como un diseño de carácter descriptivo y correlacional, no experimental, con enfoque cuantitativo. Las variables de estudio seleccionadas se agruparon en tres dimensiones: 1) la valoración del medioambiente, 2) la estimación de la gravedad de la problemática medioambiental y 3) la hipermetropía ambiental. Las variables correspondientes a la primera dimensión son las creencias y los valores que las personas presentan con respecto a la protección y cuidado del medioambiente. Por su parte, las variables de la segunda dimensión son las emociones y los sentimientos de los sujetos encuestados a la hora de percibir la problemática medioambiental. Y, finalmente, la dimensión de la hipermetropía ambiental cuenta con otras dos variables de estudio, que son la preocupación ambiental y la responsabilidad que se tiene cuando se toma conciencia del estado del ambiente a nivel local y global. La Tabla 1 muestra el conjunto de variables que se han trabajado, agrupadas en las dimensiones aquí expuestas, así como una descripción de su significado.

\section{Población y muestra}

El alumnado matriculado en las diferentes titulaciones de grado de la Facultad de Ciencias de la Educación de la Universidad de Córdoba durante el curso 2016-2017 ( $\mathrm{N}=2051$ alumnos)² se constituyó como la población objeto de estudio. El tamaño de muestra mínimo para este universo fue de 333 sujetos, según lo mostrado por Arkin y Colton (1967), asumiendo un nivel de error de un $5 \%$ en el ámbito de las Ciencias Sociales. La elección de las y los par-

2 Datos aportados por el Rectorado de la Universidad de Córdoba, sobre matriculaciones en la Facultad de Ciencias de la Educación, correspondientes al año académico 2016-2017. 
Tabla 1. Variables de estudio

\begin{tabular}{|c|c|c|}
\hline Dimensión & Definición & Variables \\
\hline $\begin{array}{l}\text { Valoración del } \\
\text { ambiente }\end{array}$ & $\begin{array}{l}\text { Grado de adhesión a creencias y valores } \\
\text { afines al cuidado y respeto del entorno, } \\
\text { así como el conjunto de sentimientos } \\
\text { de preocupación y estimación de la } \\
\text { gravedad o importancia de los diferentes } \\
\text { problemas ambientales. }\end{array}$ & $\begin{array}{l}\text { - Creencias: conjunto de conceptos, opiniones, ideas, } \\
\text { informaciones, percepciones y afirmaciones sobre la } \\
\text { naturaleza de un fenómeno (Acebal, 2010). } \\
\text { - Valores: juicios adquiridos a lo largo de nuestras vidas } \\
\text { que comenzamos heredando de personas cercanas } \\
\text { pertenecientes al entorno familiar y de instituciones } \\
\text { (Murray, 2011). }\end{array}$ \\
\hline $\begin{array}{l}\text { Estimación de } \\
\text { la gravedad } \\
\text { del problema } \\
\text { ambiental }\end{array}$ & $\begin{array}{l}\text { Conjunto de emociones y sentimientos } \\
\text { relacionados con la temática } \\
\text { medioambiental, desde la preocupación } \\
\text { por el estado del ambiente hasta su } \\
\text { percepción como medio de vida en el } \\
\text { que se puede desarrollar un sentido de } \\
\text { pertenencia y empatía con lo que nos } \\
\text { rodea (Corraliza et al., 2004). }\end{array}$ & $\begin{array}{l}\text { - Emoción: “componente esencial de nuestro modo de } \\
\text { ser y estar en el mundo en tanto influencia nuestras } \\
\text { percepciones y reacciones" (Brunel, 1995, p. 177). } \\
\text { - Sentimientos: experiencia subjetiva de la emoción. } \\
\text { "Es el estado del sujeto caracterizado por la impresión } \\
\text { afectiva que le causa determinada persona, animal, } \\
\text { cosa, recuerdo o situación en general" (Castillo, 2008, } \\
\text { p. 346). }\end{array}$ \\
\hline $\begin{array}{l}\text { Hipermetropía } \\
\text { ambiental }\end{array}$ & $\begin{array}{l}\text { Tendencia de las personas a una mayor } \\
\text { preocupación cuanto más alejado se } \\
\text { encuentre un problema ambiental, } \\
\text { al tiempo que el sentimiento de } \\
\text { responsabilidad para poder resolver estos } \\
\text { problemas disminuye por las distancias, } \\
\text { lo que aumenta los sentimientos de } \\
\text { impotencia (Uzzell, 200o). }\end{array}$ & $\begin{array}{l}\text { - Preocupación ambiental: parámetro descriptivo del } \\
\text { nivel de concienciación ambiental; es una variable } \\
\text { predictora del comportamiento ambiental (Corraliza } \\
\text { y Berenguer, 20oo). } \\
\text { - Responsabilidad ambiental: buenas conductas o } \\
\text { prácticas en lo relacionado con el ambiente y la } \\
\text { valoración del impacto ecológico de las decisiones. } \\
\text { "Conjunto de valores éticos y educativos para tomar } \\
\text { decisiones que no traigan como consecuencia la } \\
\text { afectación al ambiente. Voluntad, compromiso y } \\
\text { capacidad de contribuir al respeto y cuidado del } \\
\text { medio ambiente" (Tábara, 20o6, p. 93). }\end{array}$ \\
\hline
\end{tabular}

Fuente: Elaboración propia.

ticipantes se llevó a cabo mediante un proceso de muestreo aleatorio estratificado, teniendo en cuenta como criterios de clasificación el grado en el que se encuentra matriculado el alumnado (Educación Infantil, Educación Primaria y Educación Social) y el curso $\left(1^{\circ}\right.$ a $4^{\circ}$ en el caso de los grados en Educación Infantil y Educación Primaria y $1^{\circ}$ a $3^{\circ}$ en el caso de Educación Social). La muestra resultante ascendió a un total de 531 participantes.

Teniendo en consideración la cantidad de estudiantes, el género y su edad, hay que señalar un promedio de edad de 22 años, entre los que se encuentran 443 mujeres $(83,4 \%)$ y 88 hombres $(16,6 \%)$. En cuanto a la titulación, $38,2 \%$ está matriculado en
Educación Infantil (203 personas), 43,1\% en Educación Primaria (229 personas) y 18,6\% en Educación Social (99 personas).

\section{Instrumento}

Se ha elegido el cuestionario como instrumento de recolección de datos, pues permite la recopilación de gran cantidad de información de una manera sencilla y uniforme en un corto espacio de tiempo, de manera organizada y sistemática. El primer paso en la elaboración de la herramienta ha sido la definición de su propósito, consistente en obtener y registrar datos relativos a la dimensión afectiva de la conciencia ambiental de todas aquellas personas 
matriculadas en los diferentes grados impartidos en la Facultad de Ciencias de la Educación de la Universidad de Córdoba durante el curso 2016-2017 (Educación Infantil, Educación Primaria y Educación Social). En función de este objetivo, se han identificado y definido las variables de estudio a partir de la lectura y análisis bibliográfico de material teórico relativo a la conciencia ambiental, su conceptuación y medición.

Para medir los indicadores de creencias, valores, actitudes y comportamientos ambientales, existen numerosos instrumentos que han probado tener una adecuada confiabilidad y validez en diferentes contextos, entre los que se encuentran: la escala del Nuevo Paradigma Ecológico (NEP) propuesta por Van Liere y Dunlap (1978); diferentes escalas de preocupación ambiental, comportamiento y estructura de creencias ambientales (Maloney y Ward, 1973; Stern, 1993; Weigel y Weigel 1978); la escala de Ecocentrismo y Antropocentrismo, originalmente de Thompson y Barton (1994), adaptada al idioma español por González y Amérigo en 1999 y posteriormente complejizada por Amérigo et al. (2005); y el Cuestionario de Actitudes Ambientales de Nuévalos de 2008, a partir del cual se obtiene información respecto de la dirección e intensidad de la actitud de respeto al medio ambiente, entre otras.
Tras localizar estos constructos de medición de la conciencia ambiental en diferentes ámbitos, surgió la necesidad de diseñar un instrumento que validase las finalidades de investigación planteadas, debido a la ausencia de una herramienta específica que midiese únicamente la dimensión afectiva de la conciencia ambiental del alumnado universitario. Se construyó un banco de preguntas de acuerdo con cada dimensión de análisis, el cual configuró la estructura inicial del cuestionario, y se lo sometió a un proceso de validación de dos etapas: un juicio de expertas y expertos y una prueba piloto como procedimiento metodológico, a fin de otorgarle consistencia y solidez al instrumento de recogida de datos.

En un primer momento se trabajó con ocho expertas y expertos en materia de conciencia y educación ambiental, en función de su experiencia académica y laboral y de su profesión, buscando pluralidad territorial y equidad de género, como se muestra en la Tabla 2.

Para cuantificar el acuerdo de las juezas y de los jueces se ha utilizado el estadístico $\vee$ de Aiken, el cual ha dado como resultado un valor de 0,89 para pertinencia y de 0,79 en claridad para el instrumento en su conjunto. A partir de estos valores, así como también de las sugerencias y aportaciones realizadas, se

Tabla 2. Descripción del grupo de expertas y expertos

\begin{tabular}{|c|c|c|c|c|}
\hline Número & Sexo & Ocupación & $\begin{array}{c}\text { Unidad/ } \\
\text { Departamento }\end{array}$ & $\begin{array}{c}\text { Lugar de } \\
\text { trabajo }\end{array}$ \\
\hline 1 & Mujer & Profesora Titular Universidad & Didáctica y Organización Escolar & Cádiz \\
\hline 2 & Hombre & Personal de Administración y Servicios & Servicio de Protección Ambiental & Córdoba \\
\hline 3 & Mujer & Profesora Titular Universidad & Didáctica y Organización Escolar & Cádiz \\
\hline 4 & Mujer & Profesora Titular Universidad & Teoría e Historia de la Educación y & Sevilla \\
\hline 5 & Hombre & Profesor Ayudante Doctor & Didáctica de las Ciencias Sociales y & Córperimentales \\
\hline 6 & Hombre & Profesor Ayudante Doctor & Didáctica de las Ciencias Sociales y \\
Experimentales & Córdoba \\
\hline 7 & Hombre & Profesor Titular Universidad & Didáctica & Cádiz \\
\hline 8 & Hombre & Catedrático Escuelas Universitarias & Botánica, Ecología y Fisiología Vegetal & Córdoba \\
\hline
\end{tabular}

Fuente: Elaboración propia. 
elaboró un segundo cuestionario. Este fue sometido a una prueba piloto con alumnado de tercer año de Educación Primaria, en la que participaron un total de 61 estudiantes. Del análisis estadístico realizado para verificar la fiabilidad del estudio, se obtuvo una consistencia interna baja, con un Alfa de Cronbach de 0,345 , lo que indicó una baja fiabilidad y obligó a llevar a cabo una revisión de las medidas escalares.
Acercándonos al cálculo del poder de discriminación de los ítems (Tabla 3), de los 35 elementos escalares contenidos en las tres dimensiones del cuestionario relativas a la dimensión afectiva de la conciencia ambiental, 24 no discriminaban, lo cual representa un $68,6 \%$ y significa que los alumnos pudieron, o bien no entender las preguntas, o haber respondido al azar.

Tabla 3. Discriminación de ítems posprueba piloto

\begin{tabular}{|c|c|c|c|c|}
\hline Elementos & $\begin{array}{l}\text { Media } \\
\text { alto }\end{array}$ & $\begin{array}{l}\text { Media } \\
\text { bajo }\end{array}$ & $\mathbf{t}$ & $\mathbf{p}$ \\
\hline Los seres humanos tienen derecho a dominar al resto de la naturaleza. & 2,08 & 1,72 & $-0,899$ &, 376 \\
\hline La tierra tiene recursos limitados. & 4,33 & 3,92 & 1,064 & ,296 \\
\hline La ciencia y la tecnología podrán resolver todos los problemas medioambientales. & 2,58 & 2,44 & $-0,343$ &, 737 \\
\hline La naturaleza es sagrada y debería dejarse en paz. & 4,25 & 3,44 & $-2,302$ & ,029 \\
\hline $\begin{array}{l}\text { La resolución de los problemas medioambientales debe dejarse a expertos y } \\
\text { expertas en el tema. }\end{array}$ & 3,50 & 2,56 & $-2,252$ & ,032 \\
\hline $\begin{array}{l}\text { El ingenio humano asegurará que la tierra siga siendo un lugar habitable sin } \\
\text { cambiar nuestros hábitos. }\end{array}$ & 2,42 & 2,22 & $-0,541$ &, 593 \\
\hline El consumismo, como estilo de vida, causa y profundiza los problemas ambientales. & 4,75 & 4,17 & $-2,024$ &, 053 \\
\hline $\begin{array}{l}\text { La tierra tiene recursos naturales en abundancia; tan solo tenemos que aprender a } \\
\text { explotarlos. }\end{array}$ & 3,25 & 2,39 & $-1,875$ & , 071 \\
\hline $\begin{array}{l}\text { Es posible mantener el equilibrio ecológico (acceso a educación, trabajo, disfrute del } \\
\text { aire y agua limpios) y tener una buena calidad de vida. }\end{array}$ & 4,67 & 3,83 & $-3,038$ &, 005 \\
\hline $\begin{array}{l}\text { La capacidad de autorregulación de la naturaleza es suficiente para contrarrestar el } \\
\text { impacto de la sociedad industrializada. }\end{array}$ & 2,28 & 1,92 & 0,973 & 345 \\
\hline $\begin{array}{l}\text { El resto de las especies animales deben tener el mismo derecho a la vida que los } \\
\text { seres humanos. }\end{array}$ & 4,33 & 4,00 & 0,868 & 398 \\
\hline $\begin{array}{l}\text { Hay que boicotear a las empresas que contaminan el medioambiente y exigir } \\
\text { productos ecológicos. }\end{array}$ & 3,17 & 2,94 & $-0,553$ &, 585 \\
\hline $\begin{array}{l}\text { Cada cual, con pequeños actos cotidianos, puede hacer una contribución } \\
\text { importante a la protección del ambiente. }\end{array}$ & 4,58 & 4,44 & $-0,540$ &, 594 \\
\hline Odio a la humanidad por lo que le ha hecho a la naturaleza. & 3,08 & 2,06 & $-2,765$ & ,010 \\
\hline Me gusta ir de excursión al campo, por ejemplo, al bosque o a la montaña. & 4,58 & 4,22 & $-1,478$ & 150 \\
\hline $\begin{array}{l}\text { Los problemas medioambientales no son tan graves como se refleja en los medios } \\
\text { de comunicación. }\end{array}$ & 1,72 & 1,67 & 0,246 & ,807 \\
\hline $\begin{array}{l}\text { Si las cosas continúan como hasta ahora, pronto experimentaremos una gran } \\
\text { catástrofe ecológica. }\end{array}$ & 4,08 & 3,39 & $-1,979$ &, 058 \\
\hline $\begin{array}{l}\text { La idea de que la humanidad va a enfrentarse a una crisis ecológica global se ha } \\
\text { exagerado enormemente. }\end{array}$ & 2,83 & 2,39 & $-1,347$ & 189 \\
\hline $\begin{array}{l}\text { Las personas tienen tendencia a una visión negativa y catastrófica sobre los } \\
\text { problemas ambientales. }\end{array}$ & 3,25 & 2,72 & $-1,474$ & ,152 \\
\hline
\end{tabular}




\begin{tabular}{|c|c|c|c|c|}
\hline Elementos & $\begin{array}{c}\text { Media } \\
\text { alto }\end{array}$ & $\begin{array}{c}\text { Media } \\
\text { bajo }\end{array}$ & $\mathbf{t}$ & $\mathbf{p}$ \\
\hline $\begin{array}{l}\text { Nos estamos aproximando al número límite de personas que la tierra puede } \\
\text { albergar. }\end{array}$ & 3,67 & 2,94 & $-1,884$ & , 070 \\
\hline Los seres humanos están abusando seriamente de los recursos naturales. & 4,58 & 4,17 & $-1,751$ & 091 \\
\hline La mayoría de las actividades humanas dañan al medioambiente. & 3,67 & 3,50 & 0,412 & ,684 \\
\hline El equilibrio de la naturaleza es muy delicado y fácilmente alterable. & 4,00 & 3,39 & $-2,151$ & , O40 \\
\hline Aún estamos a tiempo de encontrar soluciones a los problemas del medioambiente. & 4,00 & 3,72 & $-0,812$ & ,424 \\
\hline Percibo la problemática ambiental como uno de los principales problemas actuales. & 4,08 & 3,67 & $-1,185$ &, 246 \\
\hline Las amenazas medioambientales no son asunto mío. & 2,25 & 1,50 & $-1,606$ &, 129 \\
\hline Pienso que son más graves los problemas globales que los locales. & 3,75 & 2,39 & $-3,618$ & , O०1 \\
\hline $\begin{array}{l}\text { Me preocupan más los problemas locales, por su cercanía y porque me afectan } \\
\text { directamente. }\end{array}$ & 3,42 & 2,39 & $-2,460$ & ,020 \\
\hline $\begin{array}{l}\text { Es muy difícil que una persona como yo pueda hacer algo por el medio ambiente a } \\
\text { nivel global. }\end{array}$ & 3,83 & 1,94 & $-5,199$ & ,0०० \\
\hline Puedo participar en la solución de los problemas medioambientales en mi ciudad. & 4,11 & 4,08 & 0,096 & ,925 \\
\hline Puedo participar en la solución de los problemas medioambientales en mi facultad. & 4,17 & 3,92 & 0,851 & 402 \\
\hline Puedo participar en la solución de los problemas medioambientales en mi hogar. & 4,58 & 4,44 & $-0,645$ &, 524 \\
\hline Me siento más responsable de los problemas locales que de los problemas globales. & 3,92 & 2,61 & $-4,231$ &, 000 \\
\hline La problemática ambiental global es muy abstracta y no me afecta directamente. & 2,67 & 1,83 & $-2,270$ & ,031 \\
\hline Me siento impotente cuando veo problemas globales en TV o internet. & 3,72 & 3,33 & 1,038 & 308 \\
\hline
\end{tabular}

Fuente: Elaboración propia.

A partir de estos datos, se tomó la decisión de mantener las preguntas tal como estaban para las variables que efectivamente discriminaban $(31,4 \%)$, reformular otras para brindar una mayor claridad en el enunciado y mejorar su comprensión, y/o suprimir algunos ítems cuando se considerase oportuno, hasta obtener el cuestionario definitivo. Este se aplicó en el mes de mayo de 2017, con la cooperación del profesorado, que permitió el ingreso a las aulas al comienzo de cada clase para que el alumnado completara la encuesta.

El instrumento presenta variables independientes (sexo, edad, titulación, curso, profesión de la madre, nivel de estudios de la madre, profesión del padre, nivel de estudios del padre y lugar de residencia) y variables dependientes que se agrupan en 32 ítems divididos en tres bloques, los cuales pretenden analizar los diferentes aspectos de la dimensión afectiva de la conciencia ambiental. La escala de respuesta de 1 a 5 indica el grado de desacuerdo o acuerdo, donde 1 significa "muy en desacuerdo", 2 "en desacuerdo", 3 "indiferente", 4 "de acuerdo" y 5 "muy de acuerdo".

ANÁLISIS DE LOS DATOS: la información procedente del cuestionario fue tratada analíticamente mediante el programa informático SPSS versión 22, realizándose pruebas de tendencia central y dispersión para comprobar el comportamiento de cada uno de los elementos que configuran cada dimensión del estudio 3 .

3 La investigación llevada a cabo ha sido más amplia y ha efectuado pruebas de contraste de hipótesis en atención a 


\section{Resultados}

Para alcanzar los resultados del estudio de la dimensión afectiva de la conciencia ambiental en el alumnado de la Facultad de Ciencias de la Educación se efectuó un análisis estadístico por bloques, asumiendo igualdad de varianzas y calculando la media y la desviación típica de cada elemento. Es de destacar que el instrumento, a partir de las correcciones realizadas luego de la prueba piloto, aumentó su fiabilidad, teniendo como resultado un Alfa de Cronbach de 0,594.

\section{Bloque 1: Valoración del ambiente}

$\mathrm{El}$ primer bloque, tendiente a medir la valoración del ambiente, arrojó como resultado que el conjunto de creencias y valores con tendencia ecocéntrica ha tenido una media igual o superior a 4 (de acuerdo y muy de acuerdo), lo que indica que el alumnado está de acuerdo y muy de acuerdo con los ítems relacionados con la sostenibilidad.

Por otro lado, se observa una media inferior a 2,66 (en desacuerdo y muy en desacuerdo) en las respuestas a los ítems que presentan una visión antropocéntrica del ambiente, según la cual el ser humano está fuera de la naturaleza y tiene total derecho de explotarla (Pato et al., 2005).

\section{Bloque 2: Estimación de la gravedad de la problemática ambiental}

En relación con el bloque 2, que analiza el grado de preocupación sobre la problemática ambiental, los datos muestran que una visión pesimista o que estima como grave la problemática tiene una media superior a 3 (entre indiferente y de acuerdo), mientras que las perspectivas optimistas o aquellas

diferentes variables predictoras, como el sexo, el curso y la titulación en la que se encuentra matriculado el alumnado, así como también pruebas de correlación para verificar la validez de las medidas encontradas. Específicamente, se realizaron pruebas de $t$ de Student para muestras independientes en función del género y análisis de varianza de un factor (ANOVA) a partir de la titulación y el curso. que restan gravedad al asunto tienen una media inferior a 3 (en desacuerdo).

\section{Bloque 3: Hipermetropía ambiental}

Finalmente, se presentan los resultados del bloque 3, el cual indaga por la hipermetropía ambiental, considerada como la tendencia a otorgarles mayor importancia a los problemas ambientales globales por sobre los locales y a sentirse, a su vez, menos responsable por la resolución de las problemáticas globales, dada la lejanía respecto de estas.

En la pregunta 40 del cuestionario, se listan una serie de 12 problemáticas ambientales, seis globales y seis locales, solicitando al alumnado enumerar las primeras tres que considere más relevantes. Los resultados indican, tal como se puede observar en la Tabla 4, que las tres problemáticas ambientales consideradas más relevantes para los encuestados han sido las globales, como el agotamiento de recur-sos, el calentamiento global y la desigualdad social.

Por otro lado, tal como se observa en la Tabla 5, a medida que las problemáticas medioambientales se vuelven más próximas y cercanas, disminuye el sen-timiento de impotencia y aumenta el sentimiento de responsabilidad para actuar e intentar resolverlas.

La Tabla 6 confirma la tendencia del alumna-do a la hipermetropía ambiental. La mayoría de las personas encuestadas está de acuerdo al percibir los problemas globales como más graves que los locales y, a la vez, en desacuerdo con la posibilidad de contri-buir en la resolución de tales problemáticas globa-les, por los niveles de dificultad que eso implicaría. Adicionalmente, a medida que se reduce el espacio de potencial actuación, esto es, ciudad-facultad-ho-gar, aumentan los sentimientos de responsabilidad en la solución de los problemas ambientales de esos contextos. Esto se demuestra a partir de la media del ítem $28(4,45)$, que afirma "Puedo participar signifi-cativamente en la solución de problemas ambienta-les en mi hogar", y, a medida que se va ampliando el espacio de acción, va bajando el grado de acuerdo. 
Tabla 4. Bloque 1: Valoración del ambiente

\begin{tabular}{|c|c|c|c|}
\hline Creencias y valores ambientales & Media & DT & $\mathbf{N}$ \\
\hline La tierra tiene recursos suficientes para satisfacer las necesidades de las nuevas generaciones. & 2,49 & 1,113 & 529 \\
\hline La ciencia y la tecnología pueden resolver todos los problemas medioambientales sin cambiar nuestros hábitos. & 2,10 & 0,936 & 528 \\
\hline La naturaleza es sagrada y debería dejarse en paz. & 4,03 & 0,876 & 523 \\
\hline La resolución de los problemas medioambientales debe dejarse a expertos y expertas del tema. & 2,65 & 1,189 & 522 \\
\hline El consumismo, como estilo de vida, causa y profundiza los problemas medioambientales. & 4,25 & 0,833 & 526 \\
\hline $\begin{array}{l}\text { Es posible mantener el equilibrio ecológico (acceso a educación, trabajo, disfrute del aire y agua limpios) y tener } \\
\text { una buena calidad de vida. }\end{array}$ & 4,03 & 0,837 & 529 \\
\hline $\begin{array}{l}\text { La naturaleza tiene una capacidad suficiente de autorregulación para compensar el impacto de la sociedad } \\
\text { industrializada. }\end{array}$ & 2,18 & 0,986 & 530 \\
\hline El ser humano es un animal superior al resto de especies animales. & 2,66 & 1,275 & 530 \\
\hline Hay que boicotear a las empresas que perjudican el medioambiente. & 3,18 & 1,057 & 524 \\
\hline $\begin{array}{l}\text { Las políticas públicas pueden contribuir más a la protección del medioambiente que nuestros pequeños actos } \\
\text { cotidianos. }\end{array}$ & 3,07 & 1,082 & 529 \\
\hline Odio a la humanidad por lo que le ha hecho a la naturaleza. & 2,62 & 1,020 & 531 \\
\hline Me gustaría pasar más tiempo en espacios naturales. & 4,17 & 0,761 & 531 \\
\hline
\end{tabular}

Fuente: Elaboración propia.

Tabla 5. Bloque 2: Estimación de la gravedad de la problemática ambiental

\begin{tabular}{|l|c|c|c|}
\hline \multicolumn{1}{|c|}{ Emociones y sentimientos ambientales } & Media & DT & N \\
\hline Actualmente estamos viviendo una gran catástrofe ecológica. & 3,89 & 0,773 & 530 \\
\hline La idea de que la humanidad va a enfrentarse a una crisis ecológica global se ha exagerado enormemente. & 2,40 & 0,950 & 530 \\
\hline Las personas tienen tendencia a una visión negativa y catastrófica sobre los problemas ambientales. & 2,95 & 0,957 & 529 \\
\hline La Tierra podrá albergar a toda la población humana, aunque esta continúe creciendo. & 2,39 & 1,008 & 526 \\
\hline El impacto de las actividades humanas en el medioambiente es negativo. & 3,83 & 0,979 & 528 \\
\hline El equilibrio de la naturaleza es muy delicado y fácilmente alterable. & 3,90 & 1,505 & 526 \\
\hline Aún estamos a tiempo de encontrar soluciones a los problemas del medioambiente. & 4,25 & 0,730 & 527 \\
\hline
\end{tabular}

Fuente: Elaboración propia.

Tabla 6. Problemáticas ambientales ordenadas en función de los mayores niveles de relevancia del alumnado

\begin{tabular}{|l|c|c|}
\hline \multicolumn{1}{|c|}{ Problemáticas } & f & \% \\
\hline Agotamiento de recursos & 332 & 62,5 \\
\hline Calentamiento global & 300 & 56,5 \\
\hline Desigualdad social & 244 & 46,0 \\
\hline Degradación de la capa de ozono & 161 & 30,3 \\
\hline Desastres naturales & 124 & 23,4 \\
\hline Despilfarro de agua & 99 & 18,6 \\
\hline Falta de espacios verdes & 58 & 10,9 \\
\hline Superpoblación humana & 50 & 9,4 \\
\hline Iluminación innecesaria & & 20 \\
\hline Despilfarro de papel & 3,8 \\
\hline Mal uso aire acondicionado/calefacción & 17 & \\
\hline Falta de contenedores para residuos & \multicolumn{2}{|c|}{3,2} \\
\hline
\end{tabular}

Fuente: Elaboración propia. 
Tabla 7. Bloque 3: Hipermetropía ambiental

\begin{tabular}{|l|c|c|c|}
\hline \multicolumn{1}{|c|}{ Preocupación y responsabilidad ambiental } & Media & DT & N \\
\hline Percibo la problemática ambiental como uno de los principales problemas actuales. & 4,01 &, 836 & 529 \\
\hline Pienso que son más graves los problemas globales que los locales. & 3,19 & 1,014 & 528 \\
\hline Me preocupan más los problemas locales, por su cercanía y porque me afectan directamente. & 2,95 &, 967 & 526 \\
\hline Es muy difícil que una persona como yo pueda hacer algo por el medio ambiente a nivel global. & 2,18 & 1,042 & 528 \\
\hline Puedo participar significativamente en la solución de los problemas medioambientales en mi ciudad. & 4,06 &, 745 & 528 \\
\hline Puedo participar significativamente en la solución de los problemas medioambientales en mi facultad. & 4,12 &, 760 & 529 \\
\hline Puedo participar significativamente en la solución de los problemas medioambientales en mi hogar. & 4,45 &, 701 & 527 \\
\hline Me siento más responsable de los problemas locales que de los problemas globales. & 3,13 & 1,052 & 527 \\
\hline La problemática ambiental global es muy abstracta y no me afecta directamente. & 2,04 &, 928 & 526 \\
\hline Cuando veo problemas medioambientales globales en TV o internet siento que no los puedo solucionar. & 3,06 &, 991 & 526 \\
\hline
\end{tabular}

Fuente: Elaboración propia.

\section{Discusión y conclusiones}

De acuerdo con los resultados obtenidos, y respondiendo al objetivo principal que ha tenido este artículo de analizar la dimensión afectiva de la conciencia ambiental en el alumnado de la Facultad de Ciencias de la Educación de la Universidad de Córdoba, se concluye que existe un significativo nivel de autopercepción de conciencia ambiental en términos de adopción de creencias y valores proambientales.

En primer lugar, se encontró que la mayoría de las personas encuestadas presenta una visión del ambiente ecocéntrica e integral, por oposición a perspectivas de carácter antropocéntrico y reduccionista, en tanto tiene mayoritariamente una percepción del ambiente integradora de las relaciones entre las personas y el entorno natural en la búsqueda del equilibrio socioambiental y la sostenibilidad (Pato et al., 2005). Aquellas personas que tienen creencias ecocéntricas serán más propensas a proteger el ambiente, mientras que aquellas con creencias antropocéntricas tenderán a dominar y explotar los recursos naturales para su satisfacción (Lui y Chen, 2020).

En segundo lugar, la mayoría considera la desigualdad social como una de las problemáticas ambientales más relevantes. Esto nos lleva a concluir que el alumnado no solo está preocu- pado por los efectos de la acción antrópica en la naturaleza y en el deterioro ambiental, sino también en las consecuencias que esa degradación ambiental produce en la sociedad. Las respuestas evidencian una representación social de los problemas ambientales y que las personas encuestadas entienden que la desigualdad, la injusticia, la discriminación de género, la pobreza e indigencia, entre otras tantas problemáticas, son parte de la realidad ambiental.

Con respecto a los niveles de preocupación y sensibilidad medioambientales del alumnado sujeto de estudio, se observó que mayoritariamente se presenta una perspectiva pesimista en relación con la gravedad de la problemática medioambiental, lo cual indica que existe un alto grado de preocupación por la misma. Se constata además que son minoritarias las perspectivas optimistas que restan trascendencia, envergadura y alcance al asunto. Esto arroja luz sobre la estimación de gravedad de la problemática ambiental por parte de los encuestados, quienes no la minimizan, al ser conscientes de la importancia que reviste en la actualidad. Este pesimismo puede caracterizarse como constructivo, en línea con la correlación entre visiones pesimistas de la realidad socioambiental futura y de comportamientos en el presente a favor del ambiente (Kaida y Kaida, 2015). 
Finalmente, se verificó la hipermetropía ambiental, entendida esta como la tendencia, por un lado, a presentar una mayor preocupación por una problemática ambiental cuanto más distante se encuentre de su realidad local, pero, por otro lado, a tener un menor sentimiento de responsabilidad para resolverla, dada la lejanía (Uzzell, 2000). Esto significa que inquietan y se priorizan más los problemas globales por sobre los locales y que han ido disminuyendo los sentimientos de responsabilidad a medida que los problemas ambientales se vuelven más abstractos o alejados del ámbito local. Al respecto, Gifford et al. denominan "optimismo espacial" la tendencia a ver las condiciones próximas como más favorables respecto de aquellas más lejanas (2009, p. 1).

Para resolver la hipermetropía ambiental es necesario pensar los problemas globales en su cotidianeidad, bajar sus niveles de abstracción e indagar sobre causas, dinámicas y efectos particulares que puedan darse en las poblaciones y entornos locales, a fin de entender que el problema supone un riesgo, pero que ese riesgo es en algún punto controlable. Si las personas pueden localizar una problemática y experimentarla, en términos concretos de afectación de su salud, su bienestar o el de la comunidad en la que viven, es más probable que lleven a cabo acciones para resolver dicha problemática (Chen, 2020).

La educación es un medio importante para generar conciencia colectiva, teórica y práctica, a favor del ambiente (Schneiderhan-Opel y Bogner, 2020). Los resultados obtenidos en esta investigación pretenden constituirse en elementos clave que sirven de diagnóstico del estado de sensibilidad ambiental existente en el alumnado universitario de la Facultad de Ciencias de la Educación, los cuales han detectado una necesidad: resolver la hipermetropía ambiental. Adicionalmente aportan información para evaluar la integración de criterios ambientales a la gestión universitaria, de acuerdo con la normativa sobre política ambiental de la Universidad de Córdoba.

Este estudio permite un acercamiento para responder a la pregunta de si la universidad funciona como promotora política de valores ambientales $y$, por ende, como multiplicadora de ellos en otros ámbitos. Pero, fundamentalmente, se trata de conocer el contenido de esos valores y los diferentes significados que las personas atribuyen al ambiente y a la cuestión socioambiental. Se intenta subrayar el impacto que las creencias y valores tienen sobre los sentimientos de preocupación y sensibilidad del alumnado en materia socioambiental, con la finalidad de generar predisposiciones para llevar adelante cambios de hábitos y de consumo más responsables con el medio. Si se percibe un riesgo -o se tiene una preocupación, en nuestro caso de estudio-, se actúa; por eso hay que medir esas percepciones. 


\section{Referencias}

Acebal, M. C. (2010). Conciencia ambiental y formación de maestras y maestros. [Tesis doctoral] Universidad de Málaga. https://libros.metabiblioteca.org/bitstream/o01/323/8/978-84-9747-606-5.pdf

Amérigo, M., Aragonés, J. I., Sevillano, V. y Cortés, B. (2005). La estructura de las creencias sobre la problemática medioambiental. Psicothema, 17(2), 257-262. http://www.psicothema.es/pdf/3096.pdf

Arkin, H. y Colton, R. (1967). Tables for Statisticians. Barnes \& Noble.

Báez, J. E. (2016). La conciencia ambiental en España a principios del siglo XXI y el impacto de la crisis económica sobre la misma. Papers, 101(3), 363-388. http://dx.doi.org/10.5565/rev/papers.2145

Brieger, S. A. (2019). Social identity and environmental concern: The importance of contextual effects. Environment and Behavior, 51(7), 828-855. https://doi.org/10.1177/0013916518756988

Brunel, M. L. (1995). La place des émotions en psychology et leur role dans le échanges conversationnels. Santé Mentale au Quebec, 20(1), 177-206. https://doi.org/10.7202/032338ar

Castillo, C. (2008). Teoría de los sentimientos. Tusquets.

Chen, M. F. (2020). Effects of psychological distance perception and psychological factors on pro-environmental behaviors in Taiwan: Application of construal level theory. International Sociology, 35(1), 70-89. https://doi. org/10.1177/0268580919881870

Chuliá, E. (1995). La conciencia ambiental de los españoles en los noventa. ASP Research Paper, 12(A), 1-36.

Corraliza, J. y Berenguer, J. (2000). Preocupación ambiental y comportamientos ecológicos. Psicothema, 12(3), 325-329. http://www.psicothema.com/psicothema.asp?id=338

Corraliza, J., Martín, R., Moreno, M. y Berenguer, J. (2004). El estudio de la conciencia ambiental. Un estudio psicosocial. En R. de Castro (coord.), Persona, sociedad y medio ambiente. Perspectivas de la investigación social de la sostenibilidad (pp. 106-120). Conserjería de Medio Ambiente, Junta de Andalucía.

Dietz, T., Stern, P. C. y Guagnano, G. (1998). Social structural and social psychological bases of environmental concern. Environment and behavior, 30, 450-471. https://doi.org/10.1177/001391659803000402

Dunlap, R. y Jones, R. (2002). Environmental concern: Conceptual and measurement issues. En R. E. Dunlap y W. Michelson (eds.), Handbook of Environmental Sociology (pp. 482-524). Greenwood Press.

Expósito, C. D. (2018). Valores básicos del profesorado: una aproximación desde el modelo axiológico de Shalom Schwartz. Educación y Educadores, 21(2), 307-325. https://doi.org/10.5294/edu.2018.21.2.7

Febles Elejalde, M., Pérez Iglesias, D. y Ramos Martínez, D. (2004). Psicología y conciencia ambientales. Un enfoque teórico para el desarrollo de la conciencia ambiental, hacia el desarrollo sostenible. Energía y Tú, 57. http://www.cubasolar.cu/57-64-2/ 
Fernández Garrido, S. y Alegre Agís, E. (2019), Cuando la voz tiembla y la disculpa incorporada emerge: autoetnografías en clave feminista. En Autoetnografías, cuerpos y emociones (II). (pp. 23-35). Universitat Rovira i Virgili.

García Linera, Á. (2017). Medioambiente e igualdad social. [Portal web] Con Nuestra América, 20 de mayo. https:// connuestraamerica.blogspot.com/2017/05/medioambiente-e-igualdad-social.html

García-Mira, R., Real, J., E. y Romay, J. (2005). Temporal and spatial dimensions in the perception of environmental problems: An investigation of the concept of environmental hyperopia. International Journal of Psychology, 40(1), 5-10. https://doi.org/10.1080/00207590444000078

García-Mira, R. y Real, J. E. (2001). Dimensiones de preocupación ambiental: una aproximación a la hipermetropía ambiental. Estudios de Psicología, 22(1), 87-96. https://doi.org/10.1174/021093901609622

Gifford, R., Scannell, L., Kormos, C. et al. (2009). Temporal pessimism and spatial optimism in environmental assessments: An 18-nation study. Journal of Environmental Psychology, 29(1), 1-12. https://doi.org/10.1016/j. jenvp.2008.06.001

Gomera, A. (2011). Análisis, medición y distribución de la conciencia ambiental en el alumnado universitario: Una herramienta para la educación ambiental. [Tesis doctoral] Universidad de Córdoba, España.

Gomera, A., Villamandos, F. y Vaquero, M. (2013). Construcción de indicadores de creencias ambientales a partir de la escala NEP. Acción Psicológica, 10(1), 149-160. http://dx.doi.org/10.5944/ap.10.1.7041

Jiménez, M. y Lafuente, R. (2010). Defining and measuring environmental consciousness. Revista Internacional de Sociología, 68(3), 731-755. https://doi.org/10.3989/ris.2008.11.03

Jiménez, M. y Lafuente, R. (2006). La operacionalización del concepto de conciencia ambiental en las encuestas. En R. Castro (ed.), Persona, sociedad y medio ambiente. Perspectivas de la investigación social de la sostenibilidad (pp. 121-150). Junta de Andalucía.

Kaida, N. y Kaida, K. (2015). Facilitating pro-environmental behavior: The role of pessimism and anthropocentric environmental values. Social Indicators Research, 126, 1243.1260. https://doi.org/10.1007/s11205-015-0943-4

Lui, W. y Chen, J. (2020). Modified two major environmental values scale for measuring Chinese children's environmental attitudes. Environmental Education Research, 26(1), 130-147. https://doi.org/10.1080/13504622.20 19.1697431

Maloney, M. P.y Ward, M. P. (1973). Ecology: Let's hear from the people. American Psychologist, 28, 583-586. https:// doi.org/10.1037/hoo34936

Manrique, P. (2019). La tercera ola revolucionaria de/contra la Modernidad. En VV.AA., Feminismos a la contra. Entre-vistas al Sur Global (pp. 13-27). La Vorágine.

Mora, E. (2019). La percepción flotante situacional. Autoetnografía y emociones. En S. Fernández y E. Alegre (eds.), Autoetnografias, cuerpos y emociones (II) (pp. 81-102). Universitat Rovira i Virgili. 
Murray, P. (2011). The sustainable self: A personal approach to sustainability education. Earthscan. https://doi. org/10.4324/9781849775212

Novo, M. (2009). La educación ambiental, una genuina educación para el desarrollo sostenible. Revista de Educación, (1 extra), 195-217. https://dialnet.unirioja.es/servlet/articulo?codigo=3019430

Ortega Egea, J. M. y García de Frutos, N. (2013). Toward consumption reduction: An environmentally motivated perspective. Psychology and Marketing, 30(8), 660-675. https://doi.org/doi: 10.1002/mar.20636

Pato C. y Tamayo A. (2006). Valores, creencias ambientales y comportamiento ecológico de activismo. Medio Ambiente y Comportamiento Humano, 7(1), 51-66. https://mach.webs.ull.es/PDFS/Vol7_1/Vol7_1_d.pdf

Pato, C., Ros, M. y Tamayo, A. (2005). Creencias y comportamiento ecológico: un estudio empírico con estudiantes brasileños. Medio Ambiente y Comportamiento Humano, 6(1), 5-22. https://mach.webs.ull.es/PDFS/ Vol6_1/VOL_6_1_b.pdf

Pisano, I. e Hidalgo, M. C. (2013). La conciencia ambiental en Andalucía, ¿qué, cuánta, quién y por qué? Revista Bilingüe de Psicología Ambiental, 10(4), 287-310. https://doi.org/10.1174/217119713807749841

Schneiderhan-Opel, J. y Bogner, F. X. (2020). The relation between knowledge acquisition and environmental values within the scope of a Biodiversity Learning Module. Sustainability, 12(2036), 2-19. https://doi. org/10.339o/su12052036

Schultz, P. W. (2001). The structure of environmental concern: Concern for self, other people, and the biosphere. Journal of Environmental Psychology, 21, 327-339. https://doi.org/10.1006/jevp.2001.0227

Schultz, P. W., Gouveia, V. V., Cameron, L., Tankha, D., Schmuck, P. y Franek, M. (2005). Values and their relationship to environmental concern and conservation behavior. Journal of Cross-Cultural Psychology, 36(4), 457-475. https://doi.org/10.1177/0022022105275962

Schwartz, S. H. y Barnea, M. (1995). Los valores en las orientaciones políticas. Aplicaciones a España, Venezuela y Méjico. Psicología Política, 11, 15-40. https://www.uv.es/garzon/psicologia politica/N11-2.pdf

Stern, P. C. (2000). Toward a coherent theory on environmentally significant behavior. Journal of Social Issues, 56, 407-424. https://doi.org/10.1111/0022-4537.00175

Stern, P. C. (1993). Value orientations, gender and environmental concern. Environment and Behavior, 25(3), 322348. https://doi.org/10.1177/0013916593255002

Stern, P. C. y Dietz, T. (1994). The value basis of environmental concern. Journal of Social Issues, 50(3), 65-84. https://doi.org/10.1111/j.1540-4560.1994.tbo2420.x

Tábara, D. (2006). Los paradigmas culturalista, cualitativo y participativo en las nuevas líneas de investigación integrada del medio ambiente y la sostenibilidad. En R. de Castro (coord.), Persona, sociedad y medio ambiente. Perspectivas de la investigación social de la sostenibilidad (pp. 83-104). Sevilla, Junta de Andalucía. 
Thompson, S. y Barton, M. (1994). Ecocentric and anthropocentric attitudes towards the environment. Journal of Environmental Psychology, 14, 149-157. https://doi.org/10.1016/S0272-4944(05)80168-9

Uzzell, D. L. (2000). The psycho-spatial dimension of global environmental problems. Journal of Environmental Psychology, 20, 307-318. https://doi.org/10.1006/jevp.2000.0175

Van Liere, K. D. y Dunlap, R. E. (1978). Environmental concern: consistency among its dimensions, conceptualizations and empirical correlates. [Paper] Annual Meeting of the Pacific Sociological Association, Spokane, Washington.

Van Liere, K. D. y Dunlap, R. E. (1980). The social bases of environmental concern: A Review of hypotheses, explanations and empirical evidence. The Public Opinion Quarterly, 44(2), 181-197. https://doi.org/10.1086/268583

Weigel, R. y Weigel, J. (1978). Environmental concern. The development of a measure. Environment and Behaviour, 10(1). https://doi.org/10.1177/0013916578101001

Zelezny, L. C. y Schultz, P. W. (2000). Promoting environmentalism. Journal of Social Issues, 56(3), 365-371. https:// doi.org/10.1111/0022-4537.00172 
